\title{
Synthesis and structural characterization of
}

\section{glucopyranosylamide films on gold}

\author{
Mridula Kadalbajoo ${ }^{1}$, Juhee Park ${ }^{1}$, Aric Opdahl ${ }^{2,3}$, Hiromi Suda ${ }^{2}$, Carolyn A. Kitchens ${ }^{2}$, Jayne C. \\ Garno $^{2,4}$, James D. Batteas, ${ }^{2,5}$, Michael J. Tarlov ${ }^{2}$, and Philip DeShong ${ }^{\text {* }}$ \\ ${ }^{1}$ Department of Chemistry and Biochemistry, University of Maryland, College Park, MD 20742 \\ ${ }^{2}$ National Institute of Standards and Technology, Gaithersburg, MD 20899 \\ ${ }^{3}$ Current Address: Department of Chemistry, University of Wisconsin, La Crosse, La Crosse, WI 54601 \\ ${ }^{4}$ Current Address: Department of Chemistry, Louisiana State University, Baton Rouge, LA 70803 \\ ${ }^{5}$ Current Address: Department of Chemistry, Texas A\&M University, College Station, TX 77842 \\ * Author to whom correspondence should be addressed, E-mail: deshong@umd.edu
}

\section{Supporting Information}

\section{Binding of Con A}

To test the effectiveness of this class of compounds for binding of glucose specific proteins, the binding of Concanavalin A (Con A) to compound 11 was measured. This was accomplished through in situ binding studies by nanografting 11 into a background matrix of 11-mercaptoundecanol (11-MUD), which functions as a resist for non-specific protein adsorption, and then exposing the nanopatterned glucospyranosalimde to Con A. This approach allows for the selectivity of binding of the Con A to the glucose nanopatterns to be directly determined from AFM images of the surface.

Grafting of compound $\mathbf{1 1}$ was carried out using a 1-5 mM solution of compound 11 in ethanol. Following grafting of compound 11 into the 11-MUD matrix, topographic AFM images showed little contrast between the heights of the two molecular systems on the surface (Figure S1A). A clear 
contrast is observed in the lateral force images however, revealing that nanografting of the glucose compound has occurred (Figure S1B). Following grafting, $1 \mathrm{mM}$ Con A in PBS buffer was then added to the AFM liquid cell and the patterned area re-imaged. The images clearly show that Con A binds specifically to the regions with the glucose moiety (Figure S1C). Topographic AFM images show that patterned feature is now $\sim 3.5 \mathrm{~nm}$ higher than the surrounding surface, consistent with the binding of a single layer of Con A (Figure S1D). ${ }^{1}$ The details of the requisite ligand density for binding will be the subject of a future study.

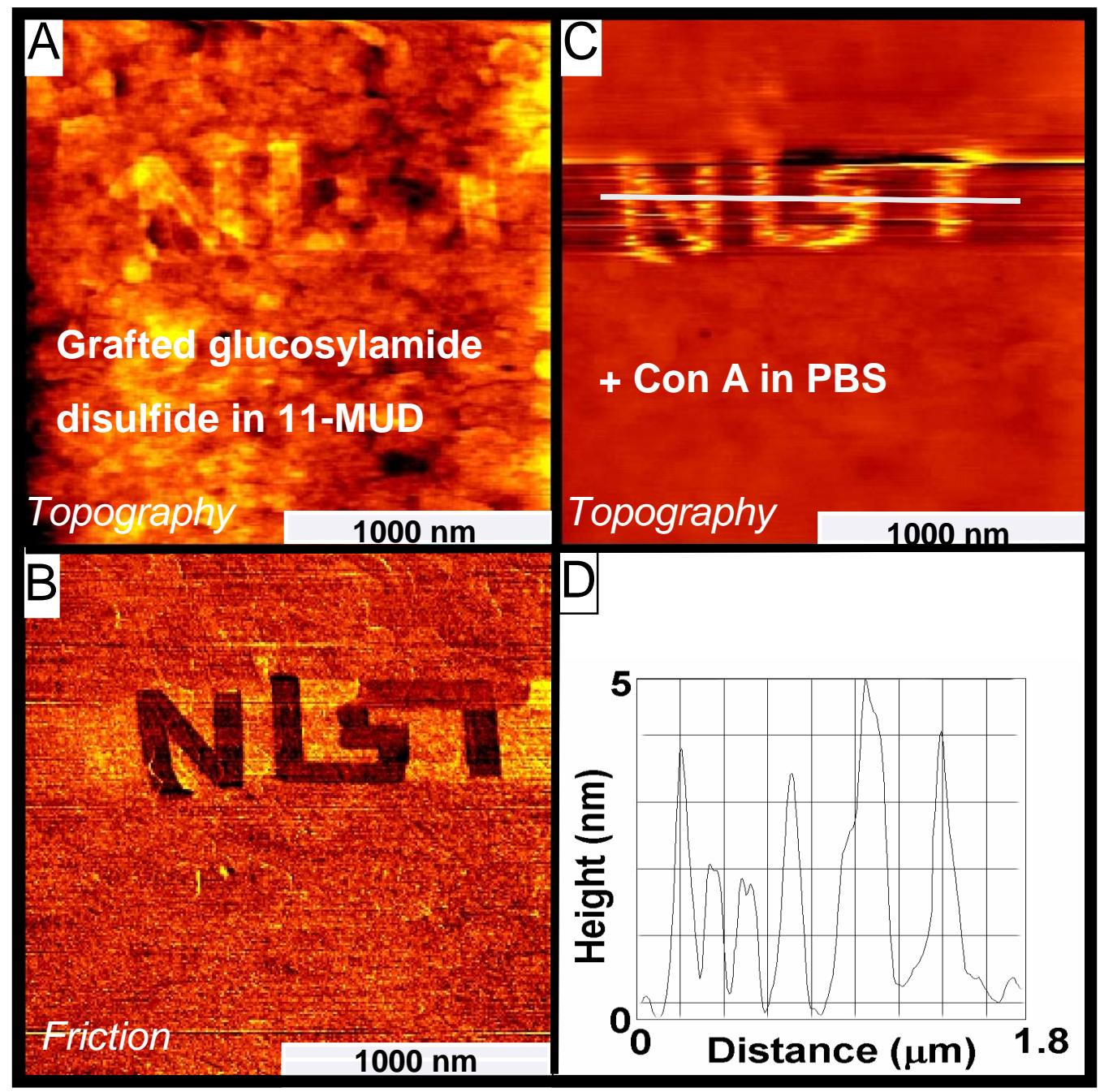

Figure S1. Binding of Con A to compound 11 nanopatterned on the Au surface. A) Topographic AFM image of compound $\mathbf{1 1}$ nanografted into a matrix of 11-mercaptoundecanol and B) the corresponding lateral force image. C) Topographic AFM image of the nanopattern following addition of Con A in PBS 
buffer. D) The line trace along the nanopattern in $\mathrm{C}$ shows that a single layer of Con $\mathrm{A}$ is bound on the pattern.

\section{Reference}

1. Waner, M. J.; Gilchrist, M.; Schindler, M. Dantus, M. J. Phys. Chem. B 1998, 102, 1649-1657. 